\title{
New structures in the continuum of light nuclei populated by two-neutron transfer reactions
}

\author{
D. Carbone ${ }^{1}$, A. Bonaccorso ${ }^{2}$, F. Cappuzzello ${ }^{1,3}$, C. Agodi $^{1}$, M. Bondi ${ }^{1,3}$, M. Cavallaro ${ }^{1}$, A. Cunsolo ${ }^{1}$, \\ M. De Napoli ${ }^{4}$, A. Foti ${ }^{1,4}$, R. Linares ${ }^{5}$, D. Nicolosi ${ }^{1,3}$, S. Tropea ${ }^{1,3}$ \\ ${ }^{1}$ INFN, Laboratori Nazionali del Sud, I-95123, Catania, Italy. \\ ${ }^{2}$ INFN, Sezione di Pisa, I-56127, Pisa, Italy \\ ${ }^{3}$ Dipartimento di Fisica e Astronomia, Università di Catania,I-95125, Catania, Italy \\ ${ }^{4}$ INFN, Sezione di Catania, I-95123, Catania, Italy \\ ${ }^{5}$ Universidade Federal Fluminense, Niteroi, Brazil
}

\begin{abstract}
The ${ }^{12} \mathrm{C}\left({ }^{18} \mathrm{O},{ }^{16} \mathrm{O}\right){ }^{14} \mathrm{C}$ reaction has been studied at $84 \mathrm{MeV}$ incident energy. The ejectiles have been momentum analysed at forward angles by the MAGNEX magnetic spectrometer. The energy spectra were obtained up to about $20 \mathrm{MeV}$ excitation energy. Calculations based on the removal of two uncorrelated neutrons from the projectile describe a significant part of the continuum observed in the energy spectra. In particular, a resonance of the residual nucleus dominates the region near the two-neutron emission threshold.
\end{abstract}

\section{Introduction}

The importance of two-neutron transfer reactions in the understanding of the pairing force has been established since many years [1]. Among these, heavy-ion direct transfer reactions at bombarding energies not much above the Coulomb barrier, have proven to be valuable tools for getting precise spectroscopic information [2] [3]. In this context, the right framework in which the reactions should be treated is a fully quantum-mechanical approach, such as Distorted Wave Born Approximation (DWBA) or Coupled Reaction Channel (CRC) methods [4], with the inclusion of the nuclear recoil. However, semi-classical approaches have proven to be accurate enough to explain integral properties such as the selectivity of the reaction, allowing also to treat the transfer to bound and unbound states in a coherent way. In particular, in ref. [5] it has been shown that different contributions to the reaction, such as elastic break-up and absorption from target bound states and resonances can be distinguished, at least for the case of one-neutron transfer.

A systematic study, aiming at the investigation of two-neutrons excitations, has been recently started at the Catania INFN-LNS laboratories exploring the $\left({ }^{18} \mathrm{O},{ }^{16} \mathrm{O}\right)$ two-neutron transfer reaction at $84 \mathrm{MeV}$ incident energy on light targets. The ${ }^{16} \mathrm{O}$ ejectiles were momentum analyzed by the MAGNEX magnetic spectrometer [6]. Thanks to the spectrometer high resolution and large acceptance, it was possible to obtain high quality inclusive spectra, even in the largely unexplored region above two-neutron emission threshold in the residual nucleus.

When dealing with transfer to the continuum, several angular momenta mix and thus the best observable to analyse is the energy distribution of the ejectile. The excitation energy spectrum of the 
residual nucleus is related to such ejectile energy through the energy conservation relation given in ref. [5]. A complete description of the measured energy spectra would require a coherent treatment of both one- and two-particle configurations for the bound and unbound systems. Moreover, the cross section should be built as a coherent sum of the sequential transfer contribution plus the direct pair transfer one. The construction of such a model is a very ambitious task, but still beyond the present status of the theory.

As a first step, in ref. [7] we made exploratory calculations for the ${ }^{13} \mathrm{C}\left({ }^{18} \mathrm{O},{ }^{16} \mathrm{O}\right){ }^{15} \mathrm{C}$ reaction assuming an uncorrelated removal of the two neutrons. The method extended the formalism of the transfer to bound states [8] to the case of unbound ones [9] [10]. In ref. [7] it is demonstrated that such calculations give a good account for the continuum background in the ${ }^{15} \mathrm{C}$ spectrum, showing an enhancement of the cross section just above the two-neutron emission threshold $\left(S_{2 n}\right)$. However, some $2 p-3 h$ resonances were not reproduced due to the lack of core polarization excitations. Here we report about the same calculations done for studying the ${ }^{14} \mathrm{C}$ continuum.

\section{Experiment and data analysis}

The ${ }^{18} \mathrm{O}^{6+}$ beam at $84 \mathrm{MeV}$ incident energy was produced and accelerated by the Tandem Van de Graaff facility of INFN-LNS. A $49 \mu \mathrm{g} / \mathrm{cm}^{2}$ self-supporting ${ }^{12} \mathrm{C}$ target was used. The ${ }^{16} \mathrm{O}$ ejectiles were momentum analysed by the MAGNEX spectrometer working in the full acceptance mode (solid angle $\Omega \sim 50 \mathrm{msr}$ and momentum range $\Delta p / p \sim 24 \%$ ). The spectrometer optical axis was located at $\theta_{\text {lab }}^{\text {opt }}=$ $12^{\circ}$, which correspond to a total covered angular range between $7.5^{\circ}$ and $17.5^{\circ}$ in the laboratory reference frame. The ejectiles were identified, event by event, by the simultaneous measurement of the position and angle at the focal plane, the energy loss in the gas section of the focal plane detector and the residual energy on the silicon hodoscope [11]. Details about the technique can be found in ref. [12]. The horizontal and vertical positions and angles at the focal plane were used as an input for a $10^{\text {th }}$ order reconstruction of the scattering angle and momentum modulus, based on the fully algebraic method implemented in MAGNEX [13]. This allows a compensation of the high order aberrations connected with the large acceptance of the spectrometer. The $Q$-values, or equivalently the excitation energy $E_{x}=Q_{0}-Q$ (where $Q_{0}$ is the ground state $Q$-value), were extracted by the application of relativistic kinematic transformations. An overall energy and angular resolution of about $160 \mathrm{keV}$ and $0.3^{\circ}$ were obtained, mainly determined by the straggling introduced in the target.

The ${ }^{12} \mathrm{C}\left({ }^{18} \mathrm{O},{ }^{16} \mathrm{O}\right){ }^{14} \mathrm{C}$ reaction is interpreted as a two-step mechanism: ${ }^{18} \mathrm{O}+{ }^{12} \mathrm{C} \rightarrow{ }^{17} \mathrm{O}+{ }^{13} \mathrm{C}_{\text {g.s. }} \rightarrow$ ${ }^{16} \mathrm{O}+{ }^{13} \mathrm{C}_{\text {g.s. }}+n$ starting from the one-neutron separation energy $\left(S_{n}=8.176 \mathrm{MeV}\right)$ and ${ }^{18} \mathrm{O}+{ }^{12} \mathrm{C} \rightarrow$ ${ }^{17} \mathrm{O}+{ }^{12} \mathrm{C}_{\text {g.s. }}+\mathrm{n} \rightarrow{ }^{16} \mathrm{O}+{ }^{12} \mathrm{C}_{\text {g.s. }}+n+n$ starting from the two-neutron separation energy $\left(S_{2 n}=13.122\right.$ $\mathrm{MeV}$ ). The continuum spectrum of ${ }^{14} \mathrm{C}$, given in Figure 1 (left panel), can be divided in three region bounded by $S_{n}$ and $S_{2 n}$. At very low $E_{x}$, the bound states of ${ }^{14} \mathrm{C}$ are populated. At higher $E_{x}$, the resonances of ${ }^{14} \mathrm{C}$ are excited up to $S_{2 n}$. Crossing such threshold, the transfer to the continuum of ${ }^{13} \mathrm{C}+$ $n$ and the transfer to the continuum of ${ }^{12} \mathrm{C}+n+n$, originated in the first step, merge together.

According to the model described in ref.s [7] [10], the transfer probability from an initial bound state of definite energy $\varepsilon_{i}$, angular momentum $l_{i}$, and $\operatorname{spin} j_{i}$ to a final continuum states of positive energy $\varepsilon_{f}$ is given by

$$
\frac{d P}{d \varepsilon_{f}}\left(j_{f}, j_{i}\right)=\sum_{j_{f}}\left(\left|1-\bar{S}_{j_{f}}\right|^{2}+1-\left|\bar{S}_{j_{f}}\right|^{2}\right) B\left(j_{f}, j_{i}\right)
$$

where $\bar{S}_{j_{f}}$ is the energy-averaged (due to the continuum conditions) and angular-momentum dependent optical model $S$-matrix, which describes the neutron-target interaction and $B\left(j_{f}, j_{i}\right)$ is the elementary transfer probability. The latter depends on the details of the initial and final states, on the energy of relative motion and on the distance of closest approach between the two nuclei, as given in ref. [10]. The calculation of the $S$-matrix, strongly related to the choice of the neutron target optical potential, is a key point of this formalism. The first term in eq. (1), proportional to $\left|1-\bar{S}_{j_{f}}\right|^{2}$, gives the neutron elastic break-up (or diffraction), while the second term proportional to $1-\left|\bar{S}_{j_{f}}\right|^{2}$, gives the neutron absorption (or stripping) by the target. 
The cross section is calculated within a semi-classical model by integrating over the core-target distances of closest approach:

$$
\frac{d \sigma_{1 n}}{d \varepsilon_{f}}=C^{2} S \int_{0}^{\infty} b d b \frac{d P(b)}{d \varepsilon_{f}} P_{e l}(b)
$$

and the total break-up cross section is obtained by integrating over the neutron final continuum energy $\varepsilon_{f}$, calculated with respect to the target. $C^{2} S$ is the spectroscopic factor of the neutron single particle initial state, $\mathrm{d} P(b) / \mathrm{d} \varepsilon_{f}$ is given in eq. (1) and $P_{e l}(b)=\left|S_{c T}\right|^{2}=\exp \left(-\ln 2 \exp \left[\left(R_{S}-b\right) / \Delta\right]\right)$ is the core survival probability in the elastic channel [14]. The strong absorption radius is defined, according to ref. [15], as $R_{S}=1.4\left(A_{P}^{1 / 3}+A_{T}^{1 / 3}\right)$ in fm and $\Delta=0.6 \mathrm{fm}$ is a diffuseness-like parameter. Although the optical potential necessary to calculate the $S$-matrix should be energy dependent, for the present calculations, as in ref. [7], a constant potential from the parametrization given in ref. [16] for $n+{ }^{12} \mathrm{C}$ and $E=10 \mathrm{MeV}$ was adopted. In fact, it was found that an energy-dependent potential would only change the overall normalization within $30 \%$. The used potential has a Woods-Saxon real volume plus a spin-orbit and surface imaginary terms. The parameters of the potential are the same used in ref. [7].

The resulting calculations are superimposed to the continuum spectrum of ${ }^{14} \mathrm{C}$ in Figure 1 (left panel). Between $S_{n}$ and $S_{2 n}$ only the elastic break-up is shown (red dashed curve), as given by the first term of eq. (1), since in this region the absorption is dominated by resonances built mainly by two neutrons plus the ${ }^{12} \mathrm{C}$ ground state, which are not included in the adopted approach. On the other hand, above the $S_{2 n}$ threshold the resonances are quite smooth and both elastic (green dashed-dotted curve) and absorption (orange dashed-dotted curve) terms were calculated.

The calculations show an enhancement of the cross section just above $S_{2 n}$, which is consistent with the experimental data, where a large structure at about $13.5 \mathrm{MeV}$ is present. Such structure is reproduced as a convolution of the peak calculated in the ${ }^{12} \mathrm{C}+n+n$ channel and the tail of the ${ }^{13} \mathrm{C}+$ $n$ elastic break-up. The main contribution comes from the absorption of the two neutrons. This means that a ${ }^{12} \mathrm{C}+n+n$ resonant configuration can account for the observed structure. On the other hand, an unknown structure at about $17 \mathrm{MeV}$ is not reproduced within this approach. This indicates that a more complete description of the ${ }^{12} \mathrm{C}+n+n$ which includes the $n-n$ correlations would be required to describe such structure.
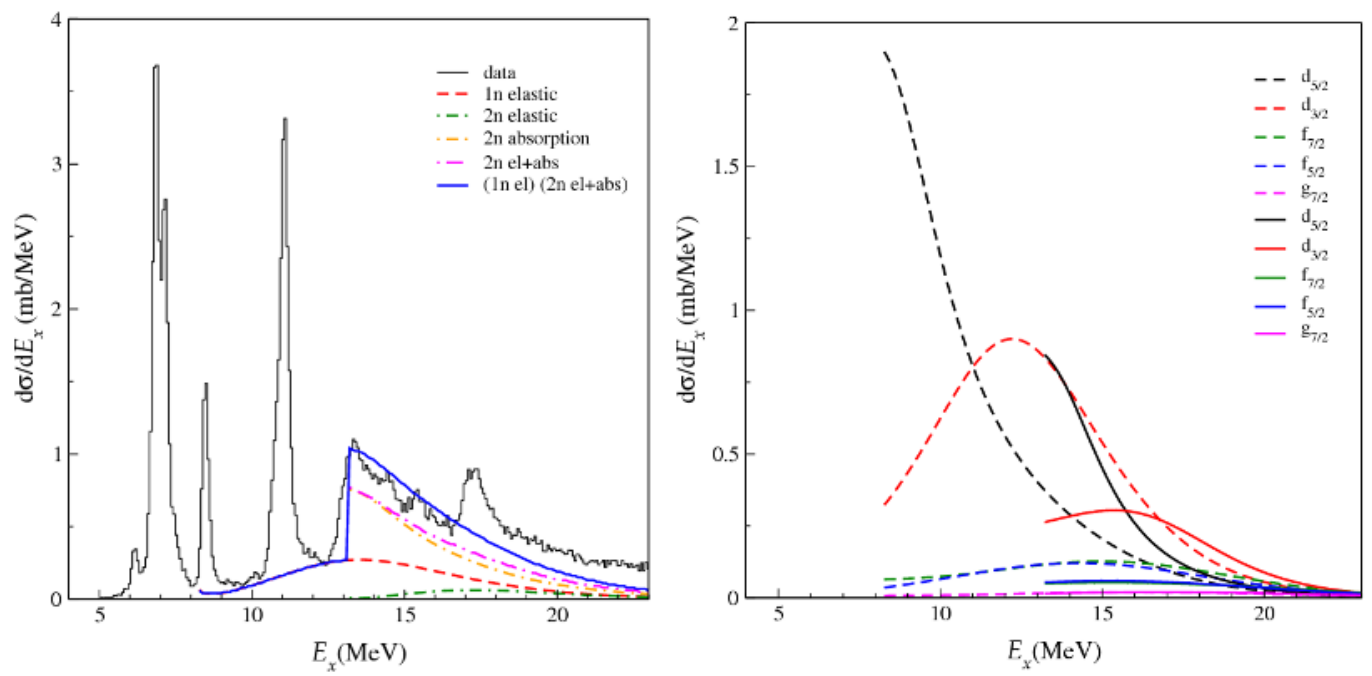

Figure 1. (left panel) Inclusive energy spectrum of the reaction ${ }^{12} \mathrm{C}\left({ }^{18} \mathrm{O},{ }^{16} \mathrm{O}\right){ }^{14} \mathrm{C}$ at $7.5^{\circ}<\theta_{\text {lab }}<17.5^{\circ}$ and theoretical calculations of various break-up components (see text): the one- (1n elastic) and two-neutron $(2 \mathrm{n}$ elastic) elastic break-up; the two-neutron absorption term ( $2 \mathrm{n}$ absorption); the sum of the two-neutron elastic and absorption terms (2n el+abs); the sum of all the contributions $((1 \mathrm{n} \mathrm{el})+(2 \mathrm{n} \mathrm{el}+\mathrm{abs}))$. (right panel) Dominant contributions to the partial wave decomposition of the theoretical energy spectrum (left panel). The legend indicates the single particle angular momentum of each individual strength distribution. The lines starting at $E_{x}=$ 8.17 MeV refer to the ${ }^{13} \mathrm{C}+n$ system while those starting at $13.12 \mathrm{MeV}$ to the ${ }^{12} \mathrm{C}+n+n$ one (see text). 
In order to understand the origin of the strength distribution in the spectrum, an estimate of the contribution of each single $l$ value to the total sum was also done. This is possible since eq. (1) contains an incoherent sum over final angular momenta. The partial wave decomposition of the $n-{ }^{13} \mathrm{C}$ elastic break-up cross sections between $S_{n}$ and $S_{2 n}$ is shown in Figure 1 (right panel). The main contribution comes from the $d_{5 / 2}$ and the $d_{3 / 2}$ states. Above $S_{2 n}$ the main source of cross section comes from configurations where both neutrons are transferred to the $d_{5 / 2}$ and the $d_{3 / 2}$ continuum orbitals, as it was found for the ${ }^{15} \mathrm{C}$ case [7].

\section{Conclusions}

In this work we have reported some results obtained studying the ${ }^{12} \mathrm{C}\left({ }^{18} \mathrm{O},{ }^{16} \mathrm{O}\right){ }^{14} \mathrm{C}$ reaction at 84 $\mathrm{MeV}$ incident energy. Using the missing mass technique, the ${ }^{16} \mathrm{O}$ ejectiles were measured by the MAGNEX and the excitation energy spectra of ${ }^{14} \mathrm{C}$ were extracted. A sudden increase of the measured ${ }^{16} \mathrm{O}$ yield is observed starting from the $S_{2 n}$ threshold, appearing in the shape of previously unobserved large structures. Both the elastic break-up and absorption channel have been analysed considering an independent removal of the two neutrons from the projectile.

The calculations give a good account for the continuum background in the ${ }^{14} \mathrm{C}$ excitation energy spectrum and show that the elastic break-up represents a minor part of the continuous spectra, which are in fact dominated by the absorption of both neutrons. In particular, an enhanced probability of exciting ${ }^{12} \mathrm{C}+n+n$ configurations near the $S_{2 n}$ threshold is obtained. The model cannot account for the strong population of the narrow resonances built mainly by two neutrons plus the target observed between $S_{n}$ and $S_{2 n}$, because of the lack of core polarization excitations. Also a structure at about 17 $\mathrm{MeV}$ is not reproduced in this approach. An explicit treatment of the full ${ }^{12} \mathrm{C}+n+n$ interaction, including the $n-n$ pairing, would be required to understand the remaining details of the energy spectra.

\section{References}

[1] D.R. Bes and R.A. Sorensen, Adv. Nucl. Phys. 2 (1969) 129.

[2] N. Anyas-Weiss et al., Phys. Reports C 12 (1974) 201.

[3] S. Kahana and A.J. Baltz, Adv. Nucl. Phys. 9 (1977) 1.

[4] G.R. Satchler, Direct Nuclear Reactions Oxford University press, Oxford (1983).

[5] A. Bonaccorso, I. Lhenry, and T. Soumijarvi, Phys. Rev. C 49 (1994) 329.

[6] F. Cappuzzello et al., in Magnets: Types, Uses and Safety (Nova Publisher Inc., NY, 2011) 1-63.

[7] F. Cappuzzello et al., Phys. Lett. B 711 (2012) 347.

[8] A. Bonaccorso, D.M. Brink, and L. Lo Monaco, J. Phys. G 13 (1987) 1407.

[9] A. Bonaccorso and D.M. Brink, Phys. Rev. C 38 (1988) 1776.

[10] A. Bonaccorso and D.M. Brink, Phys. Rev. C 43 (1991) 299.

[11] M. Cavallaro et al., Eur. Phys. J. A 48 (2012) 59.

[12] F. Cappuzzello et al., Nucl. Instr. Methods A 621 (2010) 419.

[13] F. Cappuzzello, D. Carbone, and M. Cavallaro, Nucl. Instr. Methods A 638 (2011) 74.

[14] A. Bonaccorso, Phys. Rev. C 60 (1999) 054604.

[15] D.M. Brink, Semi-Classical Methods for Nucleus-Nucleus Scattering, Cambridge University Press, 1986.

[16] J.H. Dave and C.R. Gould, Phys. Rev. C 28 (1983) 2212. 